\title{
UN FUNDAMENTO ONTOLÓGICO PARA LA PREGUNTA POR EL OTRO EN PAUL RICOEUR
}

\author{
An onotological basis for the question of the other in Paul Ricoeur
}

\author{
FEDERICO GARCÍA LARRAÍN \\ Universidad San Sebastián (Chile) \\ federico.garcia@uss.cl
}

Resumen

El supuesto de la constitución del yo a partir de la relación intersubjetiva que surge con el reconocimiento del otro hace emerger el problema del fundamento ontológico del yo. Por una parte, el yo no puede existir de manera aislada, pero por otra, subordinar su constitución a la relación con el otro puede debilitar el yo al hacerlo dependiente, para su constitución, en algo distinto de él. Hacer que la constitución del yo dependa de la relación intersubjetiva plantea la cuestión del inicio: ¿Qué son, antes de relacionarse, aquellos que entran en relación? ¿Si el yo se reconoce a sí mismo reconociendo a otro, qué es aquello que reconoce? Estas preguntas apuntan a la búsqueda de un fundamento ontológico o metafísico para la antropología fenomenológica: el uno no puede depender del otro y a su vez este del primero. La antropología filosófica de Paul Ricoeur, con su énfasis en la corporalidad e historicidad del hombre, además de su diálogo con Aristóteles, ofrece respuesta a estas preguntas. Ricoeur estudia al hombre desde dentro (a partir de su libertad), pero no olvida aquello propio del hombre que hace que el ser humano pueda ser reconocido y capaz de reconocer.

Palabras clave: Paul Ricoeur; intersubjetividad; el Otro; Fenomenología; Ontología.

\section{Abstract}

The proposition of the constitution of the self as starting from the intersubjective relationship that emerges from the recognition of the other, presents the problem of the ontological foundation of the self. On one hand, the self cannot exist in isolation, but on the other, making its constitution subordinate to the relation with the other can debilitate the self as it is made dependent, for its constitution, on something different from the self. If the constitution of the self is dependent on the intersubjective relation the question of its origin arises: What are those who enter a relation before relating? If the self only recognizes itself recognizing another, what is it that it recognizes? These questions are directed towards the search for an ontological or metaphysical foundation for the study of phenomenological anthropology. The self cannot depend on the other, and this one, in turn, on the self. Paul Ricoeur's philosophical anthropology, with its emphasis on the corporeal and historical nature of man, and its dialogue with Aristotle, offers answers to these questions. Ricoeur studies man from his inner life (beginning with his freedom) but does not forget that which makes it possible for a human being to recognize and to be recognized.

Key words: Paul Ricoeur; intersubjectivity; Otherness; Phenomenology; Ontology. 


\section{Federico García Larraín}

\section{INTRODUCCIÓN}

Dice Martin Buber en Qué es el hombre, que en la historia intelectual hay épocas en las que el hombre tiene un hogar en el mundo y otras en las que está a la intemperie. Es en estas épocas en las que el pensamiento antropológico ha sido fructífero:

Las épocas de la historia del espíritu en que le fue dado a la meditación antropológica moverse por las honduras de su experiencia fueron tiempos en que le sobrecogió al hombre el sentimiento de una soledad rigurosa, irremisible; y fue en los más solitarios donde el pensamiento se hizo fecundo. En el hielo de la soledad es cuando el hombre, implacablemente, se siente como problema, se hace cuestión de sí mismo, y como la cuestión se dirige y hace entrar en juego a lo más recóndito de sí, el hombre llega a cobrar experiencia de sí mismo (Buber, 1967, p. 24).

En las primeras, en cambio, el pensamiento antropológico es parte de la investigación más amplia acerca del mundo (cosmología) porque el hombre es parte del mundo, en armonía con él. No es que no haya una reflexión antropológica, pero es desde fuera, "en tercera persona", considerando al hombre como cosa del mundo, en un mundo de cosas. Cuando esta armonía del hombre con el cosmos se rompe, ya sea por la experiencia del mal, los descubrimientos geográficos o el cuestionamiento de lo hasta entonces sostenido, se produce una crisis. Es en estas crisis donde el hombre es forzado a encontrarse consigo mismo (Buber, 1967, pp. 26-29). Es difícil que ambas maneras de pensar al hombre se toquen o crucen; no es que se contradigan -más bien son complementarias-, pero por ocurrir en distintas épocas y surgir de experiencias distintas de la realidad, no ocurre intercambio entre ambas formas de pensar y experimentar al ser humano. Sin embargo, el filósofo hoy no puede investigar acerca del hombre sin tener en cuenta estas dos perspectivas que se han dado a lo largo de la historia.

La fenomenología es una manera de pensar al hombre en una época de crisis. El mundo moderno, dice Buber, no tiene una imagen y la generación moderna deberá vivir en un mundo no imaginable (p. 35). La fenomenología, como investigación de la realidad parte desde la experiencia y de la conciencia de esta experiencia para llegar a las cosas. Dentro de las experiencias del ser humano tiene especial relevancia la experiencia del otro como otro, que da origen a la relación de dos subjetividades.

Sin embargo, desde el punto de vista de la antropología filosófica, la salida del yo hacia el mundo, incluyendo al otro, es problemática para la fenomenología que tiende a quedarse en la experiencia subjetiva más que llegar a lo que causa la experiencia fuera del sujeto. De hecho, el mismo Husserl, hacia el final de su vida, da un giro trascendental enfocándose más en la (propia) experiencia que en la cosa en sí, que buscaba al comienzo (Smith, 2013). Esto tiene consecuencias para la constitución de yo, que queda aislado. Lo 
que comienza como una respuesta al cogito cartesiano acaba en algo muy parecido a lo que quería evitar: no es fácil superar el solipsismo.

La fenomenología se ha planteado a sí misma como una de las grandes áreas de la investigación filosófica, queda, sin embargo, plantear su relación con otras disciplinas, especialmente con la metafísica (Sánchez-Migallón, 2014). Paul Ricoeur, al entrar en diálogo directamente con René Descartes, pero tomando elementos de fuentes anteriores, como Aristóteles, y contemporáneas, como la hermenéutica y la filosofía del lenguaje, se desplaza conectando las disciplinas de la filosofía, particularmente con la metafísica. Ofrece una ontología del ser humano que permite superar el cogito cartesiano y, sacando al hombre del solipsismo, propone una intersubjetividad con fundamento. La superación del cogito en Ricoeur no es, sin embargo, una vuelta a la filosofía precartesiana (Delpech, 2011); Ricoeur asume la crisis en la que se encuentra el hombre actual, no puede pensar el hombre a la manera de Aristóteles o Tomás de Aquino, pero puede tomar elementos de ambos (y de pensadores contemporáneos, además) para lograr pensar al hombre desde dentro, pero sin comprometer su acceso al mundo externo, incluido el mundo de los otros.

\section{EL PROBLEMA DE LA INTERSUBJETIVIDAD EN LA FENOMENOLOGÍA}

En sus Meditaciones Cartesianas, Edmund Husserl busca llegar a la experiencia del otro para superar el solipsismo de Descartes. Sin embargo, la relación del yo con lo que no es yo, es problemática (Husserl, 1996, 167). Siguiendo al Descartes, Husserl pone entre paréntesis el conocimiento alcanzado hasta el momento (epoché), así llega al yo. Sin embargo, surge el peligro de caer en el solipsismo. ¿Cómo se sale del yo? ¿Es el yo lo único que existe? ¿Cómo puede el yo reconocer algo que no es él mismo? Es verdad que existen las percepciones de otras cosas, pero esas percepciones son, al fin y al cabo, parte del que percibe. Aun así, las percepciones son intencionales, es decir, dirigidas hacia afuera. Esa experiencia es una conciencia abierta pero todavía no llega al mundo.

La experiencia de lo otro aun como propio ya es reveladora, porque al ser intencional se muestra que el yo se entiende a sí mismo a partir de la experiencia de lo ajeno a sí mismo (Freud). Lo ajeno no se define por lo propio (Eigen) pero depende de lo propio. Lo otro se define por el yo, pero esta apertura no llega todavía a constituir lo otro (Husserl, 1996, p. 169).

La experiencia de lo otro como otro se constituye en la medida en que se comprende que lo otro puede ser algo como uno: se pasa del ego al alter ego, mediante la apertura al otro. Esto respeta al otro como tal y no lo incluye como parte de uno. Esta analogía parte de la experiencia primordial por la que el ego sale de sí, por lo que es trascendente. Cuando del ego se pasa al alter ego, entonces se supera el solipsismo cartesiano y a partir del otro se constituye el yo. Pero si el yo se constituye en relación con otro, cabe preguntarse cómo es que un yo puede llegar a relacionarse con otro sin estar antes constituido. Surge, además, la pregunta de cómo se reconoce a ese otro como otro 


\section{Federico García Larraín}

similar a uno. La respuesta de Husserl se hace cargo de esas dificultades: mediante la relación analógica se capta el propio cuerpo (vivido) primero y luego el cuerpo físico del otro al que se entiende como similar al propio (Husserl, 1996, p. 170).

Llegando a este punto es de interés considerar el pensamiento de Paul Ricoeur, que enfatiza el propio cuerpo con las características propias de un cuerpo material: espacialidad y temporalidad. Para Ricoeur, el cuerpo, a diferencia de lo que parece ser para Husserl, no es algo distinguible de la persona, del yo. La experiencia del yo es la experiencia del cuerpo. En esto se distancia también de Descartes, que en su duda metódica intenta separarse de su propio cuerpo. A partir de estos elementos, Ricoeur busca fundamentar una antropología filosófica que permita una real apertura hacia el otro.

\section{LA APERTURA HACIA EL OTRO EN PAUL RICOEUR}

Paul Ricoeur es un fenomenólogo en la tradición continental, pero dialoga con otras maneras de hacer filosofía, especialmente con la filosofía analítica (Dauenhauer y Pellauer, 2014). Al igual que Husserl, su punto de partida es la superación del cogito cartesiano. Sin embargo, al tomar otros elementos, distintos de la experiencia de la percepción, como el cuerpo (percepción sensible del propio yo) y con el cuerpo, el espacio, el tiempo, pasión y finitud, y por lo mismo la historia y la narrativa, rompe el solipsismo cartesiano de manera más directa. Su diálogo con Aristóteles le permite a su vez dialogar con una filosofía del hombre "desde fuera".

La obra de Ricoeur es amplísima y variadísima, sin embargo, es posible encontrar un hilo conductor en ella: se centra en el hombre y muestra la finitud del ser humano. Su filosofía es una antropología de un hombre finito, pero "capaz", que frente a sus limitaciones busca sentido (Lowe, 1981, p. 400). En este sentido, la ontología no es el primer énfasis de Ricoeur. Al respecto, dice Lowe: Ricoeur is oriented less to ontology tan to philosophical anthropology, less to the problem of truth than to the question of human meaningfulness; and between these respective issues there are important differences (p. 400). Ahora bien, el problema del significado y de la búsqueda de la trascendencia humana (399) está relacionado con el problema metafísico, porque sin valor de verdad la búsqueda del significado pierde sentido.

Paul Ricoeur comienza su obra afirmando que el hombre no es solo pensamiento. Con esto entra en discusión con la filosofía cartesiana que reduce al hombre a res cogitans -“¿Qué soy, entonces? Una cosa que piensa. Y ¿qué es una cosa que piensa? Es una cosa que duda, que entiende, que afirma, que niega, que quiere, que no quiere, que imagina también, y que siente" (Descartes, 1967, p. 227)-. No es solo pensamiento porque es cuerpo. Ahora bien, el cuerpo, que es humano, que constituye a la persona, es también fuente de sus limitaciones. El cuerpo, entre otras cosas, limita la libertad radical del ser 
humano y, afirmando esto, Ricoeur entra en polémica con Sartre, para quien la libertad es lo primero en afirmar (Lowe, 1981, p. 395).

El cuerpo es fuente de lo involuntario: de la pasión, del deseo, de lo inconsciente. Todo esto determina la voluntad, pero nunca hasta el extremo de anularla: "La libertad del hombre no es absoluta, es una libertad encarnada, síntesis de los aspectos voluntarios e involuntarios que embargan al hombre entero". La imagen de la libertad que nos da Ricoeur es la de una "libertad humana, situada, dialéctica, donde las dificultades -nunca imposibles de superar- más que del exterior, provienen de la misma condición humana" (Cuenca, 1983, p. 226). Se produce una tensión entre lo voluntario y lo involuntario. El efecto que tiene esto (la limitación del cuerpo sobre la voluntad, el efecto del inconsciente sobre el deseo) es el de producir un cogito quebrado (Ricoeur, 2008, p. XXIII). Un cogito incólume, puro espíritu, sería ilimitadamente libre, pero no sería humano. El quiebre del cogito se produce con la experiencia del cuerpo, experiencia que Descartes pone en duda, pero que no se puede dejar de lado.

El punto de interrelación con el otro comienza a producirse a partir del cuerpo. En primer lugar, es en el cuerpo donde ocurre la experiencia del mundo externo. Se puede dudar de esa experiencia, como lo hizo Descartes, pero, como nota Husserl, esa experiencia siempre está dirigida hacia algo otro, distinto del yo. En segundo lugar, el cuerpo no se origina solo. Para que exista el cuerpo son necesarios otros. En cambio, el yo cartesiano se origina en un solo acto de autoexperiencia, sin la participación de otros. En tercer lugar, el cuerpo, para vivir, necesita de la sociedad (porque el ser humano no es puro pensamiento). Esto hace que la relación con otros esté mediada por instituciones que, de alguna manera, al no depender de uno ni de otro, objetivan la relación intersubjetiva (Ricoeur, 2008, 202-203, cfr. Moratalla, 2010).

\section{LACUESTIÓN DEL OTRO EN RICOEUR}

La cuestión del otro en Ricoeur no está tanto en el reconocimiento o constitución del otro, como en el caso de Husserl, sino en el (re)conocimiento del yo a partir del reconocimiento del otro. Para Husserl primero está la experiencia del propio yo, a partir de ella se llega al otro, en un intento de superar el solipsismo del cogito. Se puede decir que Ricoeur hace el camino inverso: reconoce primero al otro, y reconociendo al otro se reconoce a sí mismo. En este sentido es revelador el título de sus conferencias dictadas con ocasión de las Gifford Lectures Sí mismo como otro. Esto ya está en cierta contraposición a Husserl (que probablemente las hubiese titulado "El otro como sí mismo").

Si bien el cuerpo es el punto de partida, el fundamento material de lo antropológico, queda por establecer cómo es que se reconoce al otro, qué es lo que se reconoce como otro, pero a la vez como propio en el otro. Es esclarecedor nuevamente Buber cuando nota que "Únicamente en la relación viva podremos reconocer inmediatamente la esencia peculiar al hombre. También el gorila es un individuo, también la termitera es una colectividad, pero 
el "yo" y el "tú" solo se dan en nuestro mundo, porque existe el hombre y el yo, ciertamente, por medio de la relación con el tú" (1967, p. 150). Las preguntas que surgen son antropológicas, propiamente tales, y también metafísicas.

¿Qué es lo que hace que un hombre sea un hombre? ¿Qué es lo que distingue al hombre del gorila, la sociedad humana del hormiguero? Se puede responder que la capacidad de relación (el hombre "capaz" ricoeuriano), pero eso no llega al fondo del problema. ¿Qué es lo que tiene el hombre que puede relacionarse con otros como él? Esa es la pregunta ontológica por el hombre. El cuerpo es insuficiente como respuesta: los gorilas y las termitas también tienen cuerpo. ¿Qué es lo propio del cuerpo humano?

Para reconocer al otro, Ricoeur busca una "idea de marca o señal por la que se reconoce" (2006, p. 20). Esa marca o señal sería lo propiamente humano. Para Aristóteles -con quien Ricoeur dialoga- esa marca sería la racionalidad (el hombre es animal racional). Para Ricoeur, en cierto sentido, también: es el cuerpo (animal) el que razona, pero además de la razón hay otros elementos que la limitan. Sin embargo, esto plantea un problema en términos ricoeurianos (no tanto para Husserl): para poder reconocer a otro por una marca o señal, hay que conocer esa señal de antemano; el sí mismo estaría, entonces, antes que el otro.

Esto se resuelve considerando que el reconocimiento del otro se logra a partir de la intencionalidad del pensamiento, del lenguaje y de la acción (Ricoeur, 2006, 113) que es anterior a una posible formulación del cogito o del yo. El yo, en Ricoeur, es un punto de llegada más que de partida porque solo actualiza sus capacidades en el encuentro con algo distinto de sí, que está fuera del yo; por lo mismo, Ricoeur prefiere usar el término "sí mismo", para distanciarse del cogito (Delgado, 2010, p. 47).

Al respecto, cobra importancia el pensamiento ético de Ricoeur como camino para una ontología del ser humano (Delgado, 2010). Se pregunta Leticia Delgado si es posible una ética sin ontología o una ontología sin ética (p. 45). El actuar humano está dirigido hacia fuera, hacia la alteridad. Como, en palabras de Delgado, no es posible una ética sin sujeto, sin libertad (p. 46), es necesaria una cierta ontología del sujeto, que Ricoeur aborda de manera tentativa hacia el final de Símismo como otro. La acción humana está dirigida hacia otro, otro que es también un sí mismo. El punto de partida para encontrarlo es la facticidad del propio cuerpo (p. 56, ver también Grassi, 2011, 2013).

La primacía del otro, de la alteridad como anterior al sí mismo se ve también al considerar al hombre en sociedad: primero -al menos temporalmente- es la sociedad, y luego el individuo. Una vez que el yo aparece, ya ha estado viviendo en sociedad, por lo que no puede considerársele aparte de ella. Esto contrasta, de nuevo, con la primacía del yo cartesiano, que se considera aparte de cualquier otro, y con propuestas éticas que hacen primar la autonomía individual por sobre cualquier consideración o vínculo anterior a la voluntad del individuo. 
La narrativa también hace que el yo retroceda a un segundo plano (y al retroceder crece en importancia el otro): la historicidad del ser humano, historicidad que depende de la temporalidad, que a su vez depende del cuerpo, implica que cuando el yo se hace consciente de sí mismo, ya lleva tiempo existiendo y no ha sido todo el tiempo el protagonista de su propia existencia, sino que ha sido hecho desde fuera, y eso incluye su propio cuerpo (Peña, 2002). Esto contrasta con diversos individualismos, en particular con el planteamiento radical de la libertad según Sartre, para quien es el sujeto el que determina lo que es.

\section{V.CONCLUSIÓN}

La ontología de la intersubjetividad comienza, para Paul Ricoeur en el hecho material del cuerpo humano, en eso tiene una semejanza con Husserl. La relación intersubjetiva se da en el encuentro con el otro, pero ese encuentro comienza en el otro, y en esto se diferencia de Husserl. Ahora bien, ese encuentro puede darse, e iniciarse en el otro porque el ser humano -el cuerpo humano- está orientado hacia fuera de sí, y una vez que encuentra a otro como sí, actualiza sus capacidades y se reconoce a sí mismo en el otro (más que al otro en sí).

La materialidad del cuerpo humano es algo fáctico, pero que permite marcar una línea directa entre la antropología del hombre que se encuentra en el mundo como en su casa, ya que el cuerpo puede tomarse como una cosa, y la antropología del hombre que está en soledad, pero que busca superarla. En este sentido, Ricoeur lleva a cabo una de las tareas pendientes de la fenomenología: se plantea las preguntas ontológicas y entrega un elemento crucial para iniciar una respuesta en diálogo con otras tradiciones (ver Bobb, 2011).

De las conclusiones alcanzadas por Ricoeur pueden derivarse varios aportes para la época actual. El quiebre del cogito cartesiano, pero sin hacerlo sucumbir al determinismo de los "maestros de la sospecha" es uno de ellos. El poner al individuo, no en un plano secundario, pero sí en un plano relativo a otros, es una manera de superar varias formas de individualismo imperante; se recuerda que el individuo, por mucho que tenga no fue capaz de darse a sí mismo dos cosas esenciales en su constitución: la existencia (que, corporalmente la recibe de otros que existían previamente) y gran parte de su identidad, que se forja en una historia en la que el individuo no es completamente protagonista. El énfasis en las limitaciones del hombre es un recordatorio de que el lugar del hombre en el mundo no es el centro: por muchas cosas que pueda dominar o controlar, nunca está en completo control de sí mismo.

Por último, la importancia dada al cuerpo como lo básicamente humano (y por lo mismo, la noción de la unidad de la persona), permiten reconocer a otros humanos más allá de cualquier factor accidental, como capacidades o facultades, desarrollo orgánico, posesiones, etc. El encuentro con otro hombre es lo que permite que uno se reconozca 
como tal: esa dependencia del otro hace que se trate al otro como a uno mismo, ya que el tratarlo como objeto o medio implica una deshumanización.

\section{OBRAS CITADAS}

Bobb, Catalin (2011). "Paul Ricoeur's hermeneutics: between epistemology and ontology". Logos \& Episteme, vol. II, no. 3: 335-345.

Buber, Martin (1967). ¿Qué es el hombre? Ciudad de México: FCE.

Cuenca, Ángel (1983). "Rasgos antropológico-éticos en el pensamiento de Paul Ricoeur". Anales de Filosofía, vol. 1: 225-240.

Dauenhauer, Bernard y Pellauer, David. 'Paul Ricoeur" (2014). The Stanford Encyclopedia of Philosophy(Summer 2014 Edition), Edward N. Zalta(ed.), URL = <http://plato.stanford.edu/archives/sum2014/entries/ricoeur/>.

Delgado, Leticia (2010). "La relación entre Ontología y Ética en Paul Ricoeur o un símismo atravesado de alteridad". Revista Laguna, № 27: 43-56.

Delpech, María Beatriz (2011). "Ricoeur y el imposible abandono total de la metafísica moderna”. IV Jornadas de Investigación en Filosofía, 27-29 noviembre de 2011, La Plata, Argentina.

Descartes, René (1967). Meditaciones Metafísicas, en Obras Escogidas, Editorial Sudamericana, Buenos Aires.

Grassi, Martín (2013). "El encuentro intersubjetivo y sus mediaciones en la fenomenología hermenéutica de Paul Ricoeur'. Tópicos, N²6: 1-20.

— (2011). "El doble acceso fenomenológico-ético a la realidad del otro en la filosofía de Paul Ricoeur'. Tópicos, № 22: 141-164.

Husserl, Edmund (1996). Meditaciones Cartesianas. Ciudad de México: FCE.

Lowe, Walter James (1981). "The Coherence of Paul Ricoeur". The Journal of Religion, vol. $61, \mathrm{~N}^{\circ} 4: 384-402$.

Moratalla, Tomás Domingo (2010). “Cuerpo Reconocido: el cuerpo en la hermenéutica del reconocimiento de Paul Ricoeur". Investigaciones Fenomenológicas, vol. monográfico 2: Cuerpo y alteridad.

Peña, Jorge (2002). La Poética del tiempo: ética y estética de la narración. Santiago: Editorial Universitaria.

Ricoeur, Paul (2008). Sí mismo como otro. Ciudad de México: Siglo XXI.

— (2006). Caminos del Reconocimiento. Ciudad de México: FCE. (2003). El conflicto de las interpretaciones. Ciudad de México: FCE. 
Sánchez-Migallón, Sergio (2014). "Fenomenología”, en Fernández, Fco. y Mercado, Juan A. (eds.). Philosophica: Enciclopedia filosófica on line, Disponible en: http://www.philosophica.info/archivo/2014/voces/fenomenologia/Fenomenologia. html

Sartre, Jean Paul (2006). El existencialismo es un humanismo. UNAM: Ciudad de México.

Smith, David Woodruff (2013). "Phenomenology", The Stanford Encyclopedia of Philosophy(Winter 2013 Edition), Edward N. Zalta (ed.). Disponible en: http://plato.stanford.edu/archives/win2013/entries/phenomenology/. 